\title{
Impact of exercise pulmonary hypertension on postoperative outcome in primary mitral regurgitation
}

\author{
Julien Magne, ${ }^{1}$ Erwan Donal, ${ }^{2,3}$ Haifa Mahjoub, ${ }^{4}$ Beatrice Miltner, ${ }^{1}$ Raluca Dulgheru, ${ }^{1}$ \\ Christophe Thebault, ${ }_{1}^{2,3}$ Luc A Pierard, ${ }^{1}$ Philippe Pibarot, ${ }^{4}$ Patrizio Lancellotti ${ }^{1}$
}

${ }^{1}$ Department of Cardiology, University of Liège Hospital, GIGA Cardiovascular Sciences, Heart Valve Clinic, CHU Sart Tilman, Liège, Belgium ${ }^{2}$ Cardiologie \& CIC-IT U 804, Centre Hospitalier Universitaire de Rennes, Rennes, France ${ }^{3}$ LTSI, Université Rennes 1, INSERM 1099, Rennes, France ${ }^{4}$ Quebec Heart and Lung Institute, Quebec, Canada

\section{Correspondence to}

Professor Patrizio Lancellotti, Department of Cardiology, University Hospital Sart Tilman, Liege B4000, Belgium; plancellotti@chu.ulg.ac.be

$\mathrm{JM}$ and ED are contributed equally to this study.

Received 3 June 2014 Revised 15 September 2014 Accepted 23 September 2014 Published Online First 17 October 2014

\section{SLinked}

- http://dx.doi.org/10.1136/ heartjnl-2014-306875

\section{CrossMark}

To cite: Magne J, Donal E, Mahjoub $\mathrm{H}$, et al. Heart 2015;101:391-396.

\section{ABSTRACT}

Aims The management of asymptomatic patients with mitral regurgitation (MR) remains controversial. Exerciseinduced pulmonary hypertension (ExPHT) was recently reported as a strong predictor of rapid onset of symptoms. We hypothesised that EXPHT is a predictor of postoperative cardiovascular events in patients with primary MR.

Methods and results One hundred and two patients with primary MR, no or mild symptoms (New York heart association (NYHA) $\leq 2$ ), and no LV dysfunction/ dilatation, were prospectively recruited in 3 centres and underwent exercise-stress echocardiography. The presence of ExPHT was defined as an exercise systolic pulmonary arterial pressure $>60 \mathrm{~mm} \mathrm{Hg}$. All patients were closely followed up and operated on when indication for surgery was reached. Postoperative events were defined as the occurrence of atrial fibrillation (AF), stroke, cardiac-related hospitalisation or death. Among the 102 patients included, 59 developed ExPHT (58\%). These patients were significantly older than those without ExPHT $(p=0.01)$. During a mean postoperative follow-up of $50 \pm 23$ months, 28 patients (26\%) experienced a predefined cardiovascular event. Patients with ExPHT had significantly higher rate of postoperative events ( $39 \%$ vs $12 \%, p=0.005)$; the rate of events was still higher in these patients ( $32 \%$ vs $9 \%, p=0.013$ ), even when excluding early postoperative $A F$ (ie, within $48 \mathrm{~h}$ ). Event-free survival was significantly lower in the ExPHT group (all events: 5 -year: $60 \pm 8 \%$ vs $88 \pm 5 \%$, $p=0.007$, events without early AF: 5 -year: $67 \pm 7 \%$ vs $90 \pm 4 \%, p=0.02$ ). Using Cox multivariable analysis, ExPHT remained independently associated with higher risk of postoperative events in all models (all $p \leq 0.04$ ).

Conclusions ExPHT is associated with increased risk of adverse cardiac events following mitral valve surgery in patients with primary MR.

\section{INTRODUCTION}

The management of asymptomatic patients with primary mitral regurgitation (MR) and no LV dysfunction or dilatation remains controversial. ${ }^{1}{ }^{2}$ In these patients, mitral valve surgery is recommended (class IIa indication, level of evidence C) in European Society of Cardiology (ESC) and American College of Cardiology and American Heart Association (ACC/AHA) guidelines in the presence of resting pulmonary hypertension (PHT) as defined as a systolic pulmonary arterial pressure (SPAP) $>50 \mathrm{~mm} \mathrm{Hg}^{3}{ }^{4}$ However, in the absence of symptoms, resting PHT is infrequent. ${ }^{5-10}$ In contrast, exercise PHT is more common in asymptomatic primary $\mathrm{MR}^{9-11}$ and was shown to be superior to resting PHT to predict the occurrence of symptoms and the need for surgery during follow-up. ${ }^{9}{ }^{10}$ Although exercise PHT is a potential or indication (ESC class IIbC) for mitral valve repair in the current guidelines (level of evidence C), there is no data in the literature regarding the postoperative outcome of patients with exercise PHT. Our group has shown that exercise PHT is independently determined by older age, resting SPAP and exercise MR severity, which are, in turn, all powerful predictors of poorer outcomes. $^{7} 8$ 12-14

Therefore, we hypothesised that exercise PHT is a predictor of postoperative cardiovascular events in patients with primary MR and no or mild symptoms.

\section{METHODS}

\section{Study population}

From July 2007 to August 2012, we prospectively included 102 consecutive patients with degenerative MR who underwent mitral valve surgery in one of the following centres: CHU Sart Tilman, Liège, Belgium $(n=36,35 \%)$, Centre Hospitalier Universitaire de Rennes, France $(\mathrm{n}=49,48 \%)$ and Quebec Heart and Lung Institute, Quebec, Canada $(\mathrm{n}=17,17 \%)$. The inclusion criteria were: (1) moderate or severe degenerative MR (defined as an effective regurgitant orifice (ERO) area $\geq 20 \mathrm{~mm}^{2}$ and/or a regurgitant volume (RV) $\geq 30 \mathrm{~mL}$ ); (2) asymptomatic or mildly symptomatic patients (New York heart association (NYHA) functional class $\leq \mathrm{II})$; (3) Preserved LVEF (>60\%) and normal LV end-systolic diameter $(<45 \mathrm{~mm})$; (4) mitral valve surgery during follow-up with class I or class IIa indication. All patients had resting and exercise Doppler-echocardiography at the time of inclusion in the study. Patients who underwent mitral valve surgery during follow-up with class IIb indication were excluded. The data from the three centres were merged in a computerised database and analysed in Liège, Belgium. All patients were in sinus rhythm and none had concomitant $>$ mild valvular stenosis or regurgitation. Patients with suspected coronary artery disease, with ST segment changes during exercise or exercise-induced wall motion abnormalities were excluded. The relevant institutional review boards approved the present protocol and all patients gave written informed consent. 


\section{Demographic and clinical data}

Demographic and clinical data included age, gender, height, weight, history of smoking, documented diagnosis of systemic hypertension, hypercholesterolaemia, overweight and diabetes.

\section{Exercise echocardiography}

Following comprehensive resting Doppler echocardiogram, all patients performed a symptom-limited graded bicycle exercise test in a semisupine position on a dedicated tilting exercise table (Ecogito, Easystress and Ergoline, 800 ER, Cosmed). The initial workload of $25 \mathrm{~W}$ was maintained for $2 \mathrm{~min}$; the workload was increased every 2 min by $25 \mathrm{~W}$. Blood pressure and a 12-lead ECG were recorded every 2 min.

\section{Echocardiographic measurements}

Echocardiographic examinations were performed with a Vivid 7 or Vivid 9 imaging device (GE Healthcare, Little Chalfont, UK). All Doppler echocardiographic data were obtained in digital format and stored on a workstation for off-line analysis (EchoPAC, GE Vingmed Ultrasound AS, Horten, Norway). All measurements were averaged over three cardiac cycles. MR was quantified as previously described ${ }^{10}$ and recommended. ${ }^{15}$ The $\mathrm{RV}$ and the ERO area were obtained. The LV diameters were obtained from the parasternal long-axis view by 2D guided $\mathrm{M}$-mode using the leading edge methodology at end-diastole and end-systole. The LV end-systolic and end-diastolic volumes as well as the LVEF were obtained using the modified biplane Simpson's method. The LV forward stroke volume was calculated by multiplying the LV outflow tract area by the LV outflow tract velocity-time integral measured by pulsed-wave Doppler. Mitral E-wave and A-wave velocities were measured with pulsed-wave Doppler, and e'-wave velocity was obtained using tissue Doppler imaging at the septal position of the mitral annulus. SPAP was derived from the regurgitant jet of tricuspid regurgitation using systolic transtricuspid pressure gradient calculated by the modified Bernoulli equation and the addition of $10 \mathrm{~mm} \mathrm{Hg}$ for right atrial pressure as previously performed ${ }^{16}$ and validated. ${ }^{10}{ }^{14}$ The right atrial pressure was assumed to be constant from rest to exercise. Resting and exercise PHT were defined as a SPAP $>50$ and SPAP $>60 \mathrm{~mm} \mathrm{Hg}$, respectively, as recommended by current guidelines. ${ }^{3} 4$

\section{Postoperative cardiac event-free survival}

The last follow-up information was obtained from interviews with the patients or physicians. Cardiac event was defined as the occurrence of postoperative cardiovascular-related death or cardiovascular-related hospitalisation, stroke or atrial fibrillation (AF). A composite end point of major postoperative cardiovascular event was defined as the occurrence of cardiovascularrelated death or cardiovascular-related hospitalisation or stroke (ie, exclusion of $\mathrm{AF}$ ). The occurrence of postoperative $\mathrm{AF}$ was separated into two phases: early AF (ie, within $48 \mathrm{~h}$ following surgery) and late postoperative AF ( $>48 \mathrm{~h})$. The follow-up collection was complete in the 102 patients $(100 \%)$.

\section{Statistical analysis}

Results are expressed as mean $\pm \mathrm{SD}$, median (25-75\% interquartile) or number (percentage) unless otherwise specified. Before analysis, normality distribution was tested with the Kolmogorov-Smirnov test. Differences between groups (ie, according to the presence or absence of exercise PHT) were analysed for statistical significance with the Student $\mathrm{t}$ test, Mann-Whitney $\mathrm{U}$ test, $\chi^{2}$ test or Fisher's exact test, when appropriate. The linear relationship between echocardiographic parameters was visually assessed using scatterplot and the coefficients of correlation reported were obtained from Pearson's or Spearman's test, as appropriate.

Probabilities of cardiac event-free survival were obtained by Kaplan-Meier estimates and then compared by the use of a twosided log-rank test. Cox proportional hazards models were used in univariable and multivariable analyses to identify the independent predictors of cardiac event-free survival. Values of $\mathrm{p}<0.05$ were considered statistically significant and all statistical tests were two-sided. All statistical analyses were performed with STATISTICA V.7 (StatSoft, Tulsa, Oklahoma, USA).

\section{RESULTS}

Among the 102 patients, 59 (58\%) developed exercise PHT. Patients with exercise PHT were significantly older and had higher prevalence of systemic hypertension (table 1). There was no other statistically significant difference between the two groups regarding demographic and clinical data, as well as resting and exercise heart rates and blood pressure. The two groups reached similar peak exercise workload (table 1). The

Table 1 Baseline demographic and clinical data according to ExPHT

\begin{tabular}{|c|c|c|c|c|}
\hline Variables & $\begin{array}{l}\text { Whole cohort } \\
n=102\end{array}$ & $\begin{array}{l}\text { No ExPHT } \\
(n=43,42 \%)\end{array}$ & $\begin{array}{l}\text { ExPHT } \\
(n=59,58 \%)\end{array}$ & p Value \\
\hline Age, years & $64 \pm 12$ & $61 \pm 13$ & $67 \pm 11$ & 0.01 \\
\hline Male, n (\%) & $69(68)$ & $29(67)$ & $41(69)$ & 0.76 \\
\hline Renal failure, $\mathrm{n}(\%)$ & $4(4)$ & $2(5)$ & $2(3)$ & 0.93 \\
\hline Systemic hypertension, $\mathrm{n}(\%)$ & $47(46)$ & $13(32)$ & $34(57)$ & 0.01 \\
\hline Diabetes, n (\%) & $7(7)$ & $2(5)$ & $5(9)$ & 0.40 \\
\hline Hypercholesterolaemia, n (\%) & $33(32)$ & $14(33)$ & $19(32)$ & 0.93 \\
\hline Resting heart rate, bpm & $72 \pm 12$ & $69 \pm 10$ & $75 \pm 13$ & 0.05 \\
\hline Resting systolic BP, mm Hg & $138 \pm 20$ & $140 \pm 20$ & $136 \pm 20$ & 0.43 \\
\hline Resting diastolic $\mathrm{BP}, \mathrm{mm} \mathrm{Hg}$ & $78 \pm 10$ & $77 \pm 11$ & $78 \pm 10$ & 0.78 \\
\hline Exercise workload, W & $80 \pm 33$ & $76 \pm 29$ & $86 \pm 37$ & 0.21 \\
\hline Exercise heart rate, bpm & $122 \pm 17$ & $121 \pm 18$ & $124 \pm 15$ & 0.46 \\
\hline Exercise systolic BP, mm Hg & $174 \pm 32$ & $173 \pm 36$ & $174 \pm 28$ & 0.92 \\
\hline Exercise diastolic BP, mm Hg & 90 [75-90] & 90 [75-90] & 89 [76.5-92] & 0.72 \\
\hline
\end{tabular}


Table 2 Baseline resting and exercise echocardiographic data according to ExPHT

\begin{tabular}{|c|c|c|c|c|}
\hline Variables & $\begin{array}{l}\text { Whole cohort } \\
n=102\end{array}$ & $\begin{array}{l}\text { No ExPHT } \\
(n=43,42 \%)\end{array}$ & $\begin{array}{l}\text { ExPHT } \\
(n=59,58 \%)\end{array}$ & p Value \\
\hline \multicolumn{5}{|l|}{ Resting data } \\
\hline LV end-systolic diameter, mm & $34 \pm 6$ & $33 \pm 6$ & $35 \pm 6$ & 0.30 \\
\hline LV end-diastolic diameter, mm & $55 \pm 8$ & $55 \pm 8$ & $56 \pm 8$ & 0.56 \\
\hline LVEF, \% & $71[66 ; 76]$ & $70[65 ; 77]$ & $71[68 ; 75]$ & 0.68 \\
\hline Regurgitant volume, $\mathrm{mL}$ & $66[53 ; 90]$ & $67[52 ; 96]$ & $65[54 ; 87]$ & 0.89 \\
\hline ERO area, $\mathrm{mm}^{2}$ & $40[35 ; 60]$ & $40[38 ; 65]$ & $40[30 ; 60]$ & 0.29 \\
\hline Left atrium surface, $\mathrm{cm}^{2}$ & $35[24 ; 76]$ & $27[20 ; 66]$ & $46[29 ; 92]$ & 0.001 \\
\hline E/e $e^{\prime}$ ratio & $14 \pm 5$ & $12 \pm 4$ & $15 \pm 6$ & 0.10 \\
\hline Systolic PAP, mm Hg & $36[30 ; 42]$ & $33[26 ; 39]$ & $40[33 ; 47]$ & 0.001 \\
\hline Severe MR, n (\%) & $83(81)$ & $35(81)$ & $48(81)$ & 0.98 \\
\hline \multicolumn{5}{|l|}{ Exercise data } \\
\hline LVEF, \% & $71 \pm 9$ & $72 \pm 9$ & $71 \pm 8$ & 0.54 \\
\hline Regurgitant volume, $\mathrm{mL}$ & $75[58 ; 98]$ & $72[46 ; 99]$ & $75[64 ; 95]$ & 0.31 \\
\hline ERO area, $\mathrm{mm}^{2}$ & $50[40 ; 73]$ & $50[40 ; 72]$ & $53[40 ; 74]$ & 0.67 \\
\hline Systolic PAP, mm Hg & $63 \pm 18$ & $47 \pm 8$ & $75 \pm 14$ & $<0.0001$ \\
\hline Severe MR, n (\%) & $83(81)$ & $31(71)$ & $52(88)$ & 0.12 \\
\hline \multicolumn{5}{|l|}{ Exercise; induced changes } \\
\hline Changes in regurgitant volume, $\mathrm{mL}$ & $+2[-9 ;+14]$ & $+1[-9 ;+10]$ & $5[-8 ;+21]$ & 0.03 \\
\hline Changes in ERO area, $\mathrm{mm}^{2}$ & $+8[0 ;+20]$ & $0[-2 ;+10]$ & $10[0 ;+25]$ & 0.02 \\
\hline Changes in systolic PAP, mm Hg & $+25[+14 ;+33]$ & $+15[+8 ;+21]$ & $+32[+28 ;+43]$ & $<0.0001$ \\
\hline
\end{tabular}

time interval between baseline exercise stress echocardiography and surgery was $2(0.5 ; 7)$ months, without significant difference between the two groups (no exercise PHT: $2(0.7 ; 9)$ months, exercise PHT: $2(0.2 ; 4)$ months, $\mathrm{p}=0.25)$. Fifty-five $(55 ; 55 \%)$ were operated on the basis of a Class I indication and $45(45 \%)$ on the basis of a Class IIa indication. Although patients with moderate $\mathrm{MR}$ at the time of baseline exercise stress echocardiography were included, all patients were operated with severe MR, according to guidelines recommendation.

\section{Echocardiographic data}

Baseline severe MR was observed in 83 (81\%) patients (table 2). The SPAP significantly increased during exercise (from 37 $\pm 11 \mathrm{~mm} \mathrm{Hg}$ to $63 \pm 18 \mathrm{~mm} \mathrm{Hg}, \quad \mathrm{p}<0.0001)$ and the exercise-induced changes in SPAP were correlated with the exercise-induced changes in RV $(r=0.41, p=0.001)$. There was a trend for weak significant correlation between the exercise-induced changes in SPAP and the changes in ERO $(\mathrm{r}=0.24, \mathrm{p}=0.07)$. As compared with patients without exercise PHT, those with exercise PHT had significantly higher resting left atrium surface $(p=0.001)$, exercise-induced increase in RV $(\mathrm{p}=0.03)$ and in ERO $(\mathrm{p}=0.02$, table 2$)$. There was no other statistically significant difference between the two groups for resting and exercise echocardiography data.

\section{Operative and postoperative outcome}

Mitral valve repair was performed in 80 (78\%) patients, without difference in the prevalence of exercise PHT (no exercise PHT: $\mathrm{n}=33,77 \%$ vs exercise PHT: $\mathrm{n}=48,81 \%, \mathrm{p}=0.59)$. Mitral valve repair was performed using Carpentier Edwards

Table 3 Distribution of total postoperative cardiovascular events according to ExPHT

\begin{tabular}{|c|c|c|c|c|}
\hline Event & Whole cohort $n=102$ & No ExPHT $(n=43,42 \%)$ & ExPHT $(n=59,58 \%)$ & p Value \\
\hline All AF, n (\%) & 19 (19) & $4(9)$ & $15(25)$ & 0.07 \\
\hline Early $A F, n(\%)$ & $5(5)$ & $1(2)$ & $4(7)$ & 0.57 \\
\hline Hospitalisation, n (\%) & $12(12)$ & $1(2)$ & $11(19)$ & 0.027 \\
\hline Acute pulmonary oedema, $\mathrm{n}(\%)$ & $2(2)$ & $0(0)$ & $2(3)$ & NA \\
\hline Stroke, n (\%) & $6(6)$ & $1(2)$ & $5(8)$ & 0.38 \\
\hline Death, $n(\%)$ & $4(4)$ & $0(0)$ & $4(7)$ & NA \\
\hline All events*, n (\%) & $28(27)$ & $5(12)$ & $23(39)$ & 0.005 \\
\hline Events without early $\mathrm{AF}^{*}, \mathrm{n}(\%)$ & $23(23)$ & $4(9)$ & $19(32)$ & 0.013 \\
\hline Major CV events*, n (\%) & $11(11)$ & $2(5)$ & $9(15)$ & 0.16 \\
\hline
\end{tabular}


Physio ring (69\%), Medtronic CG Future (29\%) and Edwards Cosgrove (2\%), without difference between the two groups. Fifty per cent of patients who undergo mitral valve replacement received a biological prosthesis, without significant difference between patients with exercise PHT and those without exercise PHT. Concomitant coronary artery bypass graft was performed in nine patients (9\%). There was no operative mortality.

During a mean postoperative follow-up of $50 \pm 23$ months (range: $10-128$ months), 28 patients (27\%) experienced a predefined cardiovascular event. All events are summarised in table 3. The first occurring events were 4 cardiovascular deaths (14\% of all events), 3 cardiac-related hospitalisations (11\%), 4 strokes (14\%), 5 early AFs (18\%) and 14 late AFs (50\%). Among the four deaths, three occurred following hospitalisation for worsening heart failure and one after fatal stroke. In these four patients, three have undergone mitral valve repair and one, mitral valve replacement. Patients with exercise PHT had a significantly higher rate of postoperative overall cardiovascular event than those without exercise PHT $(n=23,39 \%$ vs $n=5$, $12 \%, \mathrm{p}=0.005$, table 3 ). After exclusion of early AF, exercise PHT was also associated with increased rate of cardiovascular events $(n=19,32 \%$ vs $n=4,9 \%, p=0.013$, table 3 ). Among the 11 major cardiovascular events (ie, cardiac death or hospitalisation or stroke), $9(75 \%)$ occurred in the group of patients with exercise PHT $(\mathrm{p}=0.16)$.

The exclusion of patients with baseline resting PHT yielded similar results: exercise PHT remained associated with higher rate of overall postoperative cardiovascular event $(35 \%$ vs $9 \%$, $\mathrm{p}=0.002)$ and of postoperative cardiovascular event without early AF (26\% vs 6\%, p=0.01).

\section{Postoperative event-free survival}

The overall postoperative cardiac event-free survivals were 80 $\pm 4 \%, 79 \pm 4 \%, 79 \pm 4 \%$ and $71 \pm 6 \%$ at 1 year, 2 years, 3 years and 5 years, respectively. There was no significant difference in postoperative 5 -year cardiac event-free survival between mitral valve repair and mitral valve replacement $(p=0.50)$. Patients with or without resting PHT had statistically similar postoperative event-free survival (overall events: $p=0.91$; events without early AF: $p=0.48$ ). On the other hand, patients with

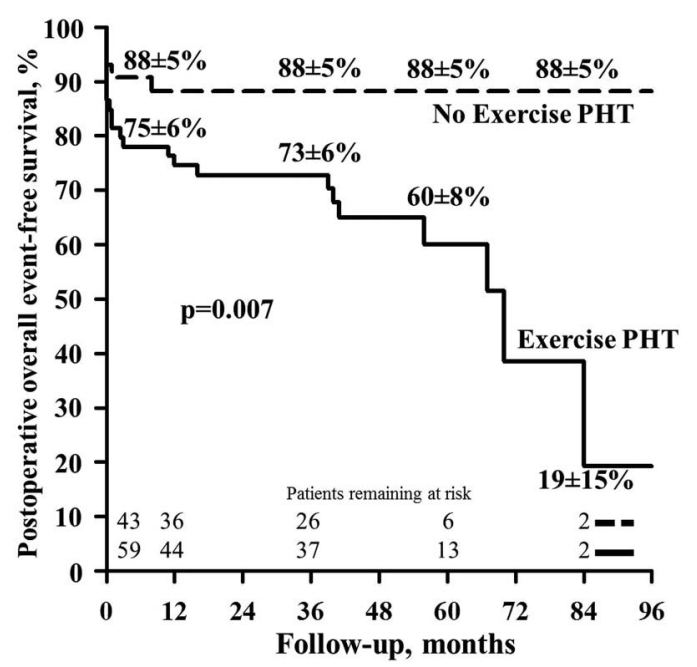

Figure 1 Postoperative overall event-free survival according to the presence or absence of exercise pulmonary hypertension (PHT). Percentages reported are the survival rates at $1-, 3-, 5-$, and 7 -year of follow-up.

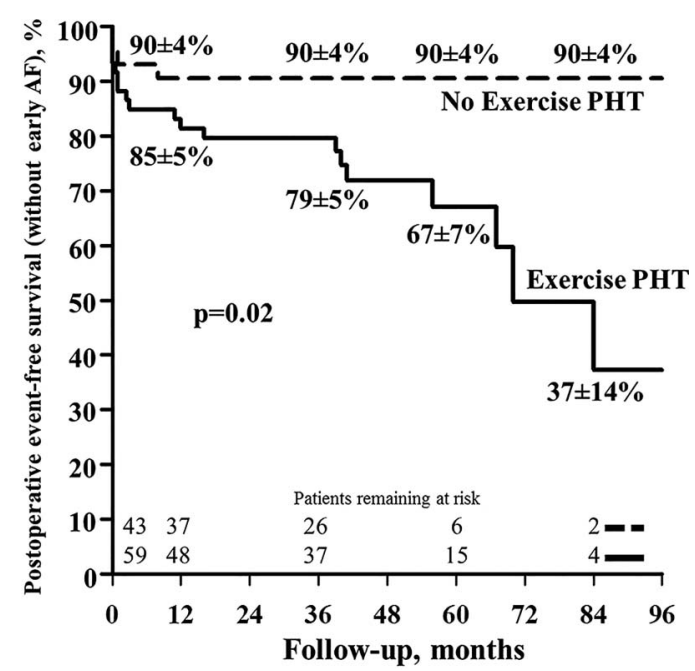

Figure 2 Postoperative event-free survival without early atrial fibrillation (AF) according to the presence or absence of exercise pulmonary hypertension (PHT). Percentages reported are the survival rates at 1-, 3-, 5-, and 7-year of follow-up.

exercise PHT had markedly lower event-free survival than those without exercise PHT (1-year: $75 \pm 6$ vs $88 \pm 5 \%$, 3-year: $73 \pm 6$ vs $88 \pm 5 \%$, 5 -year: $60 \pm 8 \%$, vs $88 \pm 5 \%, \mathrm{p}=0.007$, figure 1 ). The exclusion of early AF provided similar results with significantly lower event-free survival in the exercise PHT group (1-year: $83 \pm 5$ vs $90 \pm 4 \%, 3$-year: $79 \pm 5$ vs $90 \pm 4 \%, 5$-year: 67 $\pm 7 \%$, vs $90 \pm 4 \%, \mathrm{p}=0.02$, figure 2 ).

When patients with baseline resting PHT were excluded, exercise PHT remained significantly associated with lower overall event-free survival and event-free survival without early $\mathrm{AF}$ (1-year: $78 \pm 6$ vs $91 \pm 4 \%$, 3-year: $74 \pm 6$ vs $91 \pm 4 \%$, 5 -year: 60 $\pm 8 \%$, vs $91 \pm 4 \%, \mathrm{p}=0.004$ and 1 -year: $85 \pm 5$ vs $93 \pm 4 \%$, 3 -year: $81 \pm 5$ vs $93 \pm 4 \%, \quad 5$-year: $70 \pm 8 \%$, vs $93 \pm 5 \%$, $\mathrm{p}=0.024$, respectively).

Using Cox proportional hazards model, exercise PHT was associated with a 1.9-fold increased risk of overall postoperative events $(p=0.0096)$ and 1.8 -fold increased risk of postoperative events without early AF ( $p=0.017$, table 4). After adjustment for age and sex, exercise PHT remained significantly associated with postoperative cardiovascular event (overall: $\mathrm{p}=0.0045$, without early AF: $p=0.008$ ). Further adjustment for the parameters used as class I indication for surgery in the most recent ESC and ACC/AHA guidelines (ie, LV end-systolic diameter, LVEF and baseline NYHA functional class) yielded to significant association between preoperative exercise PHT and postoperative overall events (all $\mathrm{p} \leq 0.01$ ) and events without early AF (all $\mathrm{p} \leq 0.05$ ), respectively (table 4 ).

Similarly, after adjustment for age, sex, LVEF and preoperative NYHA functional class (ie, at the time of surgery), exercise PHT remained associated with a significantly higher risk of worse outcome (table 4).

\section{DISCUSSION}

The present study confirms that resting PHT is uncommon (9\%) in asymptomatic or mildly symptomatic patients with primary $\mathrm{MR}$, preserved LV function and no LV dilatation. In contrast, exercise PHT is more frequent (58\%) and represents a major determinant of postoperative cardiac event-free survival. Even after adjustment for parameters related to class I indication for surgery of the most recent guidelines, exercise PHT remains associated with a twofold increased risk of postoperative event. 
Table 4 Impact of exercise pulmonary hypertension on cardiac event- free survival: Cox proportional hazards regression analysis

\begin{tabular}{|c|c|c|c|c|c|c|}
\hline \multirow[b]{2}{*}{ Analysis } & \multicolumn{3}{|c|}{ All CV events } & \multicolumn{3}{|c|}{ CV events without early AF } \\
\hline & HR & $95 \% \mathrm{Cl}$ & $\mathrm{p}$ Value & HR & $95 \% \mathrm{Cl}$ & p Value \\
\hline Univariable & 1.9 & 1.1 to 3.4 & 0.0096 & 1.8 & 1.1 to 3.3 & 0.017 \\
\hline Age and sex adjusted & 2.2 & 1.2 to 4.5 & 0.0045 & 2.1 & 1.2 to 4.5 & 0.008 \\
\hline Age, sex and LV end systolic diameter adjusted & 2.2 & 1.2 to 4.5 & 0.0043 & 2.1 & 1.2 to 4.3 & 0.008 \\
\hline Age, sex and LVEF* adjusted & 2.2 & 1.3 to 4.8 & 0.0031 & 2.0 & 1.1 to 4.3 & 0.013 \\
\hline Age, sex, LVEF and baseline NYHA class adjusted & 2.0 & 1.2 to 4.3 & 0.01 & 1.8 & 1.0 to 3.7 & 0.04 \\
\hline Age, sex, LVEF and preoperative NYHA class adjusted & 2.0 & 1.2 to 1.8 & 0.0041 & 2.0 & 1.2 to 4.3 & 0.0087 \\
\hline
\end{tabular}

\section{Pathophysiology of exercise PHT}

In contrast with resting PHT, which is mainly determined by estimated LV filing pressure and left atrial area, exercise PHT more frequently occurs in patients with dynamic MR exhibiting a marked exercise-induced increase in MR severity. ${ }^{10}{ }^{11}$ During exercise, the abrupt increase in MR severity, even if mild, may exceed in some patients the level of left atrial compliance and pulmonary vascular recruitment and leads to an increase in SPAP up to PHT. Although initially benign with no significant consequences, such a phenomenon, when repetitive, can progressively conduct to left atrial dilatation, increased LV filling pressure, AF, low-level exercise dyspnoea, and finally, resting symptoms. ${ }^{17}$ In our study, the most common postoperative event was AF, which is consistent with the larger left atrial volume in patients exhibiting exercise PHT. The presence of a dynamic MR could thus be the precursor of a cascade of harmful pathophysiological abnormalities, starting with increase in left atrial dimension and exercise PHT and leading to rapid development of symptoms and higher risk of postoperative cardiac events.

\section{Postoperative outcome and exercise PHT}

The most frequent postoperative outcome reported in this multicentre study was AF (including early and late occurrence). One could argue that postoperative AF is not a 'hard' event and that this result emphasises that exercise PHT is a benign condition. However, AF in patients with primary MR is known to be associated with worse outcome and increased morbidity and mortality when conservatively managed. ${ }^{18}$ The high rate of postoperative AF, while likely related to left atrial enlargement and older age, underlines that patients with exercise PHT are probably at a more advanced stage of the disease. Hence, exercise PHT, as assessed using exercise echocardiography, could be a reasonable trigger for surgery in asymptomatic patients.

In patients with functional MR, changes in MR during exercise were also associated with changes in $\operatorname{SPAP}^{19}$ and are involved in the pathogenesis of acute pulmonary oedema. ${ }^{20}$ Although rare in primary MR, the two episodes of acute pulmonary oedema observed in our series occurred in patients with exercise PHT.

The four deaths in the whole cohort were in the group of preoperative exercise PHT. The lack of statistical comparison precludes meaningful conclusion regarding these data. Nevertheless, from a clinical standpoint, these results strongly suggest that preoperative exercise PHT is a powerful independent predictor of postoperative outcome (table 4).

As opposed to exercise PHT, resting PHT was not associated with higher risk of cardiovascular events during follow-up. This result is contrasting with current literature ${ }^{7}$ but is probably related to the small number $(n=9)$ of patients with resting PHT in our study and important differences in inclusion criteria.

\section{Clinical implication}

Our group reported in 2010 that patients with exercise PHT are more likely to rapidly develop symptoms during follow-up. This finding was further confirmed by larger series. ${ }^{9}$ The present data now reveals that preoperative exercise PHT predicts a 2 -fold increase in the risk of postoperative cardiovascular event, regardless of the symptomatic status. Of note, the vast majority of our patients were conservatively managed up to the first occurrence of class I or class IIa indications for surgery. Consequently, our results suggest that this strategy recommended by the guidelines may not be suitable for all patients, and particularly in those with exercise PHT is not adequate and could be improved. Indeed, early elective surgery in a highvolume centre with high heart valve surgery experience seems more appropriate in these patients since, when operated for other indications than exercise PHT, their postoperative outcome is significantly worse. Early surgery appears safe and associated with high postoperative survival in primary MR. ${ }^{21}$ Nonetheless, it does not seem reasonable to generalise an 'early surgery' strategy to all asymptomatic patients. Indeed, operative mortality rate is not null and the risk to perform mitral valve replacement instead of valve repair exists. ${ }^{1}$ In contrast, conservative management could be proposed to patients without exercise PHT, and a follow-up in a heart valve clinic could be recommended. ${ }^{22}$ Furthermore, this strategy could also be improved by the use of resting and exercise brain natriuretic peptide 2324 and the assessment of LV longitudinal function ${ }^{24}$ and contractile reserve. $^{25}$

In our cohort, 11 patients (58\%) with moderate MR developed exercise PHT. In accordance with current ESC and ACC/ AHA guidelines which do not recommend surgery in patients with moderate MR, except when other cardiac surgery is indicated (Class IIa, evidence C), we believe that patients with moderate MR and exercise PHT should be followed very closely and should undergo surgery as soon as they develop severe MR.

\section{Limitations}

The present series is relatively small and the number of postoperative events is limited. This could lead to type II error. However, the fact that exercise PHT was significantly associated with reduced postoperative event-free survival suggests that this relationship is strong. Furthermore, this is one of the largest studies with this design and including such patients.

The surgical procedure was not standardised in this multicentre study and depended on the surgeon preference. In this 
regard, some uncontrolled confounding surgical factors may have impacted the postoperative course. Nevertheless, this could not influence the main findings of this study.

Due to the small number of patients with resting PHT in this study $(n=9)$, we did not perform any comparison of this subset versus patients with no resting PHT and no exercise PHT and versus patients with no resting PHT and exercise PHT.

\section{CONCLUSION}

In patients with primary MR, no or mild symptoms, and no LV dysfunction/dilatation, the development of exercise PHT is a major risk factor for cardiovascular events following subsequent mitral valve surgery, regardless of symptom onset during the preoperative course. The results of this study suggest that asymptomatic patients with exercise PHT may benefit from early elective surgical intervention as suggested by current guidelines, or should, at least, be referred to heart valve clinic and be closely follow-up. Furthermore, these data emphasise the usefulness of exercise stress echocardiography in asymptomatic patients with primary MR.

\section{Key messages}

\section{What is known on this subject?}

Patients with chronic primary mitral regurgitation and who develop exercise pulmonary hypertension are at higher risk of frequent and rapid development of symptoms during the follow-up.

\section{What might this study add?}

This study shows that exercise pulmonary hypertension in chronic primary mitral regurgitation is associated with reduced postoperative cardiac event-free survival and multiplies by 2 the risk of postoperative event, even after adjustment for parameters considered as trigger for surgery in current guidelines.

\section{How might this impact on clinical practice?}

These data emphasise the usefulness of exercise stress echocardiography in patients with primary mitral regurgitation. It also demonstrates that exercise stress echocardiography and the assessment of exercise pulmonary hypertension may identify a group of patients at high risk of postoperative cardiac event.

Acknowledgements The authors thank Carmine Celentano, Jacinthe Aubé, Isabelle Fortin, Marie Guinoiseau and Valérie Le Moal for excellent technical assistance.

Contributors JM: study design, echocardiographic exams analysis, clinical data and follow-up recording, data analysis and drafting of the manuscript. ED: echocardiographic examination recording and analysis, clinical data and follow-up recording and review of manuscript. HM: echocardiographic examination recording and analysis, clinical data and follow-up recording and review of manuscript. RD: echocardiographic examination recording and analysis and review of manuscript. CT: review of the manuscript. LAP: echocardiographic examination recording and analysis, clinical data and follow-up recording and review of the manuscript. PP: study design and review of the manuscript. PL: study design, echocardiographic examination recording and analysis and review of the manuscript.

Funding This study was supported by a grant (MOP \# 102737) from the Canadian Institutes of Health Research, Ottawa, Ontario, Canada.

Competing interests PP holds the Canada Research Chair in Valvular Heart Diseases, Canadian Institutes of Health Research, Ottawa, Ontario, Canada. CT received a grant from the Fédération Française de Cardiologie.

Ethics approval Institutional Review Boards.

Provenance and peer review Not commissioned; externally peer reviewed.

\section{REFERENCES}

1 Gillam LD, Schwartz A. Primum non nocere: the case for watchful waiting in asymptomatic "severe" degenerative mitral regurgitation. Circulation 2010;121:813-21.

2 Enriquez-Sarano M, Sundt TM III. Early surgery is recommended for mitral regurgitation. Circulation 2010;121:804-11.

3 Nishimura RA, Otto CM, Bonow RO, et al. 2014 AHA/ACC Guideline for the management of patients with valvular heart disease: a report of the American College of Cardiology/American Heart Association Task Force on Practice Guidelines. Circulation 2014;129:2440-92.

4 Vahanian A, Alfieri O, Andreotti F, et al. Guidelines on the management of valvular heart disease (version 2012): the Joint Task Force on the Management of Valvular Heart Disease of the European Society of Cardiology (ESC) and the European Association for Cardio-Thoracic Surgery (EACTS). Eur Heart J 2012;33:2451-96.

5 Alexopoulos D, Lazam C, Borrico S, et al. Isolated chronic mitral regurgitation with preserved systolic left ventricular function and severe pulmonary hypertension. J Am Coll Cardiol 1989;14:319-22.

6 Yang H, Davidson WR Jr, Chambers CE, et al. Preoperative pulmonary hypertension is associated with postoperative left ventricular dysfunction in chronic organic mitral regurgitation: an echocardiographic and hemodynamic study. J Am Soc Echocardiogr 2006;19:1051-5.

7 Barbieri A, Bursi F, Grigioni F, et al. Prognostic and therapeutic implications of pulmonary hypertension complicating degenerative mitral regurgitation due to flail leaflet: a multicenter long-term international study. Eur Heart $J$ 2011;32:751-9.

8 Le Tourneau T, Richardson M, Juthier F, et al. Echocardiography predictors and prognostic value of pulmonary artery systolic pressure in chronic organic mitral regurgitation. Heart 2010;96:1311-17.

9 Kusunose K, Popovic ZB, Motoki H, et al. Prognostic significance of exercise induced right ventricular dysfunction in asymptomatic degenerative mitral regurgitation. Circ Cardiovasc Imaging 2013;6:167-76.

10 Magne J, Lancellotti P, Pierard LA. Exercise pulmonary hypertension in asymptomatic degenerative mitral regurgitation. Circulation 2010;122:33-41.

11 Magne J, Lancellotti $P, O^{\prime}$ Connor K, et al. Prediction of exercise pulmonary hypertension in asymptomatic degenerative mitral regurgitation. J Am SoC Echocardiogr 2011;24:1004-12.

12 Enriquez-Sarano M, Tajik AJ, Schaff HV, et al. Echocardiographic prediction of survival after surgical correction of organic mitral regurgitation. Circulation 1994;90:830-7.

13 Stoddard MF, Prince CR, Dillon $\mathrm{S}$, et al. Exercise-induced mitral regurgitation is a predictor of morbid events in subjects with mitral valve prolapse. J Am Coll Cardiol 1995;25:693-9.

14 Magne J, Lancellotti P, Pierard LA. Exercise-induced changes in degenerative mitral regurgitation. J Am Coll Cardiol 2010;56:300-9.

15 Lancellotti P, Moura L, Pierard LA, et al. European Association of Echocardiography recommendations for the assessment of valvular regurgitation. Part 2: mitral and tricuspid regurgitation (native valve disease). Eur J Echocardiogr 2010;11:307-32.

16 Schwammenthal E, Vered Z, Agranat O, et al. Impact of atrioventricular compliance on pulmonary artery pressure in mitral stenosis: an exercise echocardiographic study. Circulation 2000;102:2378-84.

17 Lewis GD, Bossone $E$, Naeije R, et al. Pulmonary vascular hemodynamic response to exercise in cardiopulmonary diseases. Circulation 2013;128:1470-9.

18 Grigioni F, Avierinos JF, Ling LH, et al. Atrial fibrillation complicating the course of degenerative mitral regurgitation: determinants and long-term outcome. J Am Coll Cardiol 2002;40:84-92.

19 Tumminello G, Lancellotti $\mathrm{P}$, Lempereur $\mathrm{M}$, et al. Determinants of pulmonary artery hypertension at rest and during exercise in patients with heart failure. Eur Heart $J$ 2007;28:569-74.

20 Pierard LA, Lancellotti P. The role of ischemic mitral regurgitation in the pathogenesis of acute pulmonary edema. N Engl J Med 2004;351:1627-34.

21 Kang DH, Kim JH, Rim JH, et al. Comparison of early surgery versus conventional treatment in asymptomatic severe mitral regurgitation. Circulation 2009;119:797-804.

22 Lancellotti P, Rosenhek R, Pibarot P, et al. ESC Working Group on Valvular Heart Disease position paper-heart valve clinics: organization, structure, and experiences. Eur Heart J 2013;34:1597-606.

23 Magne J, Mahjoub H, Pibarot $P$, et al. Prognostic importance of exercise brain natriuretic peptide in asymptomatic degenerative mitral regurgitation. Eur J Heart Fail 2012;14:1293-302.

24 Magne J, Mahjoub H, Pierard LA, et al. Prognostic importance of brain natriuretic peptide and left ventricular longitudinal function in asymptomatic degenerative mitral regurgitation. Heart 2012;98:584-91.

25 Magne J, Mahjoub H, Dulgheru R, et al. Left ventricular contractile reserve in asymptomatic primary mitral regurgitation. Eur Heart J 2014;35:1608-16. 


\section{Heart}

Impact of exercise pulmonary hypertension on postoperative outcome in primary mitral regurgitation

Julien Magne, Erwan Donal, Haifa Mahjoub, Beatrice Miltner, Raluca

Dulgheru, Christophe Thebault, Luc A Pierard, Philippe Pibarot and

Patrizio Lancellotti

Heart2015 101: $391-396$ originally published online October 17, 2014

doi: 10.1136/heartjnl-2014-306296

Updated information and services can be found at:

http://heart.bmj.com/content/101/5/391

References This article cites 25 articles, 11 of which you can access for free at: http://heart.bmj.com/content/101/5/391\#ref-list-1

Email alerting Receive free email alerts when new articles cite this article. Sign up in the service box at the top right corner of the online article.

Topic Articles on similar topics can be found in the following collections

Collections

Drugs: cardiovascular system (8839)

Hypertension (3004)

Mitral valve disease (229)

Clinical diagnostic tests (4778)

Echocardiography (2126)

\section{Notes}

To request permissions go to:

http://group.bmj.com/group/rights-licensing/permissions

To order reprints go to:

http://journals.bmj.com/cgi/reprintform

To subscribe to BMJ go to:

http://group.bmj.com/subscribe/ 